\section{Eflornithine Is Safer than Melarsoprol for the Treatment of Second-Stage Trypanosoma brucei gambiense Human African Trypanosomiasis}

\author{
François Chappuis, ${ }^{1,2}$ Nitya Udayraj, ${ }^{1}$ Kai Stietenroth, ${ }^{1}$ Ann Meussen, ${ }^{1}$ \\ and Patrick A. Bovier ${ }^{2}$ \\ ${ }^{1}$ Médecins Sans Frontières and ${ }^{2}$ Department of Community Medicine, \\ Geneva University Hospitals, Geneva, Switzerland
}

Patients with second-stage human African trypanosomiasis treated with eflornithine $(n=251)$ in 2003 in Kiri, southern Sudan, had an adjusted relative risk of death of 0.2 and experienced significantly fewer cutaneous and neurological adverse effects than did patients who were treated with melarsoprol in 2001 and $2002(n=708)$.

Human African trypanosomiasis (HAT) due to Trypanosoma brucei gambiense remains a significant public health problem in some of the poorest countries of sub-Saharan Africa. Melarsoprol, an arsenical drug that has been used since 1949, is still used for treating second-stage (or meningo-encephaliticstage) HAT in most areas of endemicity. This drug is highly toxic, with acute reactive encephalopathy occurring in 5\%-10\% of treated patients, resulting in a case-fatality rate of $\sim 50 \%$ [1]. Moreover, a dramatic increase in the rate of failure of treatment has been noted in several endemic foci [2].

Eflornithine (difluoromethylornithine [DFMO]), an inhibitor of ornithine decarboxylase developed in the 1980s, is the only other drug registered for the treatment of second-stage HAT. When it is administered at the recommended intravenous dose of $400 \mathrm{mg} / \mathrm{kg}$ per day for 14 days, the limited published data have shown that is has a high efficacy and is associated with case-fatality rates of $2 \%-3 \%[3,4]$. Although the drug was initially unavailable because of irregular production and high pricing, a recent 5-year donation agreement between the World Health Organization (WHO) and Aventis Pharma has made it widely available for use in the field.

Received 14 February 2005; accepted 8 April 2005; electronically published 19 July 2005 Reprints or correspondence: François Chappuis, Travel and Migration Medicine Unit, Dept. of Community Medicine, Geneva University Hospital, rue Micheli-du-Crest 24, 1211 Geneva 14, Switzerland (francois.chappuis@hcuge.ch).

Clinical Infectious Diseases 2005; 41:748-51

(C) 2005 by the Infectious Diseases Society of America. All rights reserved. $1058-4838 / 2005 / 4105-0025 \$ 15.00$
At the Médecins Sans Frontières sleeping sickness treatment center in Kiri, Kajo-Keji County, southern Sudan, patients with second-stage HAT were treated with intravenous melarsoprol at a dosage of $2.2 \mathrm{mg} / \mathrm{kg}$ per day and oral prednisone for 10 days from June 2000 to December 2002. This treatment was changed in January 2003 to a course of intravenous DFMO administered at a dosage of $400 \mathrm{mg} / \mathrm{kg}$ per day divided into four 3-h infusions for 14 days. Because of decreased bioavailability of DFMO for children aged $<12$ years [5], the daily dose was increased to $600 \mathrm{mg} / \mathrm{kg}$ for this age category. Follow-up visits were scheduled at 6,12 , and 24 months after the end of treatment. We retrospectively reviewed outcomes during this period, to compare case-fatality rates, adverse-effects, and the efficacy of melarsoprol and DFMO.

Diagnosis and clinical staging followed a methodology that has been described elsewhere [6]. Personal, laboratory, and treatment data, including adverse effects and outcomes, were recorded on standardized patients' charts and entered into YoTryp (Microsoft), a Microsoft Access-based software specifically designed to monitor the program. Most adverse effects were graded as benign to moderate (grade 1) or severe (grade 2). All patients' charts were reviewed, and errors were corrected by one of the authors (F.C.) during several field visits.

A total of 1376 patients with second-stage HAT were admitted between June 2000 and December 2003. Three hundred twenty-four patients who were treated during the initial phase of the project (June 2000-December 2000) were excluded from analysis because they were at a more advanced stage of disease, which resulted in a higher case-fatality rate. An additional 93 patients were also excluded from analysis: 46 patients who had been previously treated for second-stage illness, 15 who had been screened but not treated, and 32 who had been treated with pentamidine or other drugs. Of the 959 remaining patients, we compared the characteristics of 708 patients treated with melarsoprol in 2001 and 2002 with characteristics of the 251 patients treated with DFMO in 2003.

Baseline characteristics such as sex, residential status, mode of screening, presence of cervical lymph nodes, and CSF WBC count differed between the 2 cohorts (table 1). Twenty-five patients $(3.5 \%)$ died during melarsoprol treatment (because of acute reactive encephalopathy in $92 \%$ of cases), whereas only 2 patients $(0.8 \%)$ who were treated with DFMO died, which is a significant difference (table 2). On the basis of the number of treatment days before death, we used Cox proportional hazard regression models to adjust for differences in baseline characteristics. After adjusting for sex, residential status, mode of 
Table 1. Baseline characteristics of patients with second-stage human African trypanosomiasis treated with difluoromethylornithine (DFMO) and meIarsoprol in Kiri, Kajo-Keji County, southern Sudan.

\begin{tabular}{|c|c|c|c|}
\hline Characteristic, by class & $\begin{array}{c}\text { DFMO } \\
(n=251)\end{array}$ & $\begin{array}{l}\text { Melarsoprol } \\
(n=708)\end{array}$ & $P$ \\
\hline \multicolumn{4}{|l|}{ General } \\
\hline Age & & & .59 \\
\hline Mean $\pm \mathrm{SD}$ & $25 \pm 15$ & $25 \pm 15$ & \\
\hline Range & $1-75$ & $0.5-85$ & \\
\hline Sex & & & $<.001$ \\
\hline Male & $161(64)$ & $346(49)$ & $\ldots$ \\
\hline Female & $90(36)$ & $362(51)$ & $\ldots$ \\
\hline Residential status & & & $<.001$ \\
\hline Resident of Kajo-Keji County & $158(63)$ & $477(67)$ & $\ldots$ \\
\hline Resident of other Sudanese county & $36(14)$ & $24(3)$ & $\ldots$ \\
\hline Displaced Sudanese & 48 (19) & $167(24)$ & $\ldots$ \\
\hline Migrant Sudanese & $9(4)$ & $34(5)$ & $\ldots$ \\
\hline Non-Sudanese and/or unknown & 0 & $6(1)$ & $\ldots$ \\
\hline Mode of screening & & & $<.001$ \\
\hline Passive & $118(47)$ & $526(74)$ & $\ldots$ \\
\hline Active & $133(53)$ & $182(26)$ & $\ldots$ \\
\hline \multicolumn{4}{|l|}{ Laboratory } \\
\hline Presence of a cervical lymph node & $145(58)$ & $359(51)$ & .047 \\
\hline Positive LN puncture examination finding & $132(91)$ & $315(88)$ & .29 \\
\hline Blood concentration technique $^{a}$ & & & .11 \\
\hline Positive & $83(33)$ & $260(37)$ & $\ldots$ \\
\hline Negative & $36(14)$ & $128(18)$ & $\ldots$ \\
\hline Not done & $132(53)$ & $320(45)$ & $\ldots$ \\
\hline Trypanosomes in CSF & 99 (39) & $297(42)$ & .49 \\
\hline CSF WBC count, cells $/ \mu \mathrm{L}$ & & & .031 \\
\hline $3-19$ & $63(25)$ & $188(27)$ & $\ldots$ \\
\hline $20-99$ & $73(29)$ & $259(37)$ & $\ldots$ \\
\hline$\geqslant 100$ & $115(46)$ & $261(37)$ & $\ldots$ \\
\hline Diagnostic classification & & & .48 \\
\hline No previous treatment & $244(98)$ & $678(96)$ & $\ldots$ \\
\hline Relapse after first stage of treatment & $6(2)$ & $28(4)$ & $\ldots$ \\
\hline
\end{tabular}

screening, presence of cervical lymph nodes, and CSF cell count, the relative risk of death following DFMO treatment was 0.20 (95\% CI, 0.04-0.89).

Acute reactive encephalopathy occurred in 80 patients $(11.3 \%)$ treated with melarsoprol, but in only 1 patient $(0.4 \%)$ treated with DFMO. Moreover, patients treated with melarsoprol were more likely to suffer from fever, hypertension, macular rash, severe headaches, peripheral neuropathy, and tremor. Diarrhea was more frequent in the cohort treated with DFMO, but the occurrence of severe diarrhea among patients in both cohorts was comparable. Simple convulsions occurred in 7 patients $(2.8 \%)$ treated with DFMO. Hemoglobin and WBC counts were measured both before and at the end of treatment in a subset of 184 patients treated with DFMO. We found no statistical differences between pretreatment and posttreatment hemoglobin levels (pretreatment median, $11.7 \mathrm{~g} / \mathrm{dL}$ [range, 6.7$18.3 \mathrm{~g} / \mathrm{dL}$ ]; posttreatment median, $11.7 \mathrm{~g} / \mathrm{dL}$ [range, 6-15.3 $\mathrm{g} / \mathrm{dL}] ; P=.96$ ) and WBC counts (pretreatment median, 7350 cells $/ \mathrm{mm}^{3}$ [range, $3300-21,000$ cells $/ \mathrm{mm}^{3}$ ]; posttreatment median, 7450 cells $\mathrm{mm}^{3}$ [range, 2900-28,500 cells $/ \mathrm{mm}^{3}$ ]; $P=$ $.98)$.

The rates of patient attendance to posttreatment follow-up visits were $64 \%$ at 6 months after treatment and $46 \%$ at 12 months after treatment. There were 9 relapses $(3.6 \%)$ and 1 
Table 2. Comparison of outcomes for patients with secondstage human African trypanosomiasis treated with difluoromethylornithine and melarsoprol in Kiri, Kajo-Keji County, southern Sudan.

\begin{tabular}{|c|c|c|c|}
\hline Characteristic & $\begin{array}{l}\text { DFMO } \\
\text { recipients } \\
(n=251)\end{array}$ & $\begin{array}{l}\text { Melarsoprol } \\
\text { recipients } \\
(n=708)\end{array}$ & $P$ \\
\hline Mortality during treatment & $2(0.8)$ & $25(3.5)$ & .024 \\
\hline \multicolumn{4}{|l|}{ Adverse effects of treatment } \\
\hline Fever & $27(10.8)$ & 218 (32.3) & $<.001$ \\
\hline Hypotension & $0(0.0)$ & $6(0.9)$ & .13 \\
\hline \multicolumn{4}{|l|}{ Hypertension } \\
\hline All & $8(3.2)$ & $48(7.3)$ & .02 \\
\hline Severe $^{a}$ & $0(0.0)$ & $17(2.4)$ & .013 \\
\hline \multicolumn{4}{|l|}{ Macular rash } \\
\hline All & $1(0.4)$ & $49(7.5)$ & $<.001$ \\
\hline Severe ${ }^{b}$ & $1(0.4)$ & $18(2.5)$ & .036 \\
\hline Bullous rash & $0(0.0)$ & $4(0.6)$ & .22 \\
\hline Jaundice & $0(0.0)$ & $8(1.2)$ & .08 \\
\hline \multicolumn{4}{|l|}{ Diarrhea } \\
\hline All & $52(20.9)$ & $68(10.3)$ & $<.001$ \\
\hline Severe ${ }^{c}$ & $4(1.6)$ & $18(2.5)$ & .39 \\
\hline \multicolumn{4}{|l|}{ Headaches } \\
\hline All & $105(42.2)$ & $270(39.7)$ & .5 \\
\hline Severe ${ }^{b}$ & $0(0.0)$ & $111(15.7)$ & $<.001$ \\
\hline \multicolumn{4}{|l|}{ Peripheral neuropathy } \\
\hline All & $5(2)$ & $78(11.8)$ & $<.001$ \\
\hline Severe $^{d}$ & $0(0.0)$ & $12(1.7)$ & .038 \\
\hline \multicolumn{4}{|l|}{ Tremor } \\
\hline All & $6(2.4)$ & $149(22.7)$ & $<.001$ \\
\hline Severe $e^{e}$ & $0(0.0)$ & $18(2.5)$ & .011 \\
\hline \multicolumn{4}{|c|}{ Acute reactive encephalopathy ${ }^{\dagger}$} \\
\hline All & $1(0.4)$ & $80(11.3)$ & $<.001$ \\
\hline Grade 1 & $0(0.0)$ & $21(3)$ & .006 \\
\hline Grade 2 & $1(0.4)$ & $59(8.3)$ & $<.001$ \\
\hline
\end{tabular}

NOTE. Data shown are no. (\%) of patients, unless otherwise indicated.

a Severity is defined as systolic blood pressure of $>180 \mathrm{~mm} \mathrm{Hg}$ and/or diastolic blood pressure of $>100 \mathrm{~mm} \mathrm{Hg}$.

${ }^{b}$ Severity is defined as an event leading to interruption of treatment.

${ }^{c}$ Severity is defined as bloody diarrhea and/or signs of dehydration.

d Severity is defined as an event leading to motor weakness, walking disability, and/or interruption of treatment.

e Severity is defined as an event impairing simple activities.

f Grade 1 acute reactive encephalopathy is defined as new onset of psychosis during treatment. Grade 2 acute reactive encephalopathy is defined as prolonged or repeated convulsions (status epilepticus) or rapid deterioration of the state of consciousness (with a Glasgow Coma Score of $\leqslant 8$ ).

death $(0.4 \%)$ in the DFMO group $(n=249)$, and there were 16 relapses $(2.3 \%)$ and 6 deaths $(0.9 \%)$ in the melarsoprol group ( $n=683$ ) during the 12-month follow-up period. Posttreatment relapse and death rates after treatment with DFMO and melarsoprol were statistically comparable $(P=.29$ and $P=.46$, respectively).

This retrospective analysis shows that 14 days of therapy with intravenous DFMO resulted in fewer deaths and severe adverse effects than did 10 days of therapy with intravenous melarsoprol for the treatment of second-stage HAT. Moreover, we found no evidence of increased death or relapse rates within 12 months after the end of DFMO treatment.

The nonrandomized nature of this study, despite statistical adjustment for differences in baseline characteristics, could preclude any definitive conclusion. However, we believe our results are valid. A fairly large number of patients were analyzed, and all were treated in comparable conditions. Secondly, risk of death was minimized in the melarsoprol cohort by excluding patients who had been treated during the first 7 months of the program. Moreover, the proportion of patients with a CSF WBC count of $>100$ cells $/ \mu \mathrm{L}$, a marker of both disease severity and increased risk of treatment failure $[3,7,8]$, was higher in the DFMO cohort.

The case-fatality rate for the DFMO cohort is the lowest reported in the literature among patients treated for secondstage HAT. This excellent safety record has been confirmed in other treatment centers in southern Sudan, where DFMO is used as first-line treatment of second-stage illness. Of 3178 patients treated by Malteser (Yei County) and Médecins Sans Frontières (Mundri, Maridi and Kajo-Keji Counties) between September 2001 and December 2004, the overall case-fatality rate was $1 \%$. Melarsoprol-induced acute reactive encephalopathy is a tragedy that generally takes patients and care-givers by surprise and cannot be efficiently prevented. In contrast, severe catheter-related bacterial infections, which are frequently reported as a cause of complications in patients treated with DFMO [3], were efficiently prevented in our program by providing adequate nursing care, such as the systematic changing of intravenous catheters every $48 \mathrm{~h}$, the use of sterile gauzes around catheter puncture wounds, the application of singleuse sterile caps between infusions, and the daily surveillance and early treatment of phlebitis and deep tissue infections [9]. After this improved nursing protocol was introduced in April 2003 for patients treated with DFMO, the incidences of phlebitis and deep-tissue bacterial infection decreased from $43 \%$ to $6.3 \%(P<.001)$ and from $1.9 \%$ to $0.7 \%(P=.4)$, respectively.

On the basis of these results, we recommend DFMO as the treatment of choice for second-stage HAT, not only in areas where treatment with melarsoprol has high failure rates (i.e., where it is currently the only option), but in all endemic areas where adequate nursing care can be provided. Although the lack of trained medical staff in many remote health care centers and the insufficient financial and technical logistic capacities in most countries of endemicity represent obstacles for the universal use of DFMO, we observed that the increased nursing workload caused by treatment with DFMO (e.g., the preparation of infusions and the management of catheters) was at least partially compensated by the decreased amount of work 
required to manage adverse effects due to treatment with melarsoprol.

Nevertheless, a 14-day course of intravenous DFMO monotherapy is not a long-term solution. This regimen is complicated to administer, it is potentially less effective in areas with a high prevalence of HIV infection (DFMO is trypanostatic), and it could lead to the development of parasite resistance. In the short term, existing drugs should be studied in combination to simplify treatment and shorten its duration and to protect against resistance [10]. To this end, a multicenter study of a shorter course of intravenous DFMO administered twice per day with a course of oral nifurtimox is underway. Although this regimen is suboptimal, it remains the only possible approach, because the research pipeline for new drugs against second-stage HAT is empty. Even the future of DFMO is fragile, given that the donation agreement between the WHO and Aventis Pharma will soon expire. DFMO production must be ensured, and the development of new drugs must be promoted for the treatment of this neglected and devastating sickness.

\section{Acknowledgments}

We thank Nathan Ford from Médecins Sans Frontières in the United Kingdom for his constructive review of this manuscript.

Potential conflicts of interest. All authors: no conflicts.

\section{References}

1. World Health Organization. Control and surveillance of African trypanosomiasis: report of a WHO expert committee. WHO technical report series 881. Geneva: World Health Organization, 1998.

2. Brun R, Schumacher R, Schmid C, Kunz C, Burri C. The phenomenon of treatment failures in human African trypanosomiasis. Trop Med Int Health 2001; 6:906-14.

3. Pépin J, Khonde N, Maiso F, et al. Short-course eflornithine in Gambian trypanosomiasis: a multicentre randomized controlled trial. Bull WHO 2000; 78:1284-95.

4. Milord F, Pépin J, Loko L, Ethier L, Mpia B. Efficacy and toxicity of eflornithine for treatment of Trypanosoma brucei gambiense sleeping sickness. Lancet 1992;340:652-5.

5. Milord F, Loko L, Ethier L, Mpia B, Pépin J. Eflornithine concentration in serum and cerebrospinal fluid of 63 patients treated for Trypanosoma brucei gambiense sleeping sickness. Trans Roy Soc Trop Med Hyg 1993; 87:473-7.

6. Chappuis F, Pittet A, Bovier PA, et al. Field evaluation of the CATT/ Trypanosoma brucei gambiense on blood-impregnated filter papers for diagnosis of human African trypanosomiasis in southern Sudan. Trop Med Int Health 2002; 11:942-8.

7. Schmid C, Nkunku S, Merolle A, Vounatsou P, Burri C. Efficacy of 10-day melarsoprol schedule 2 years after treatment for late-stage gambiense sleeping sickness. Lancet 2004;364:789-90.

8. Pépin J. African trypanosomiasis. In: Strickland GT, eds. Hunter's tropical medicine and emerging infectious diseases. 8th ed. Philadelphia: W.B. Saunders, 2000:643-53.

9. Chappuis F. Eflornithine for the treatment of human African trypanosomiasis, practical perspectives. Développement et Santé 2004; 171: $41-7$.

10. Legros D, Ollivier G, Gastellu-Etchegorry M, et al. Treatment of human African trypanosomiasis-present situation and needs for research and development. Lancet Infect Dis 2002;2:437-40. 